\title{
A Comparison of Refractive Accuracy Between Conventional and Femtosecond Laser Cataract Surgery Techniques Using Modern IOL Formulas
}

This article was published in the following Dove Press journal: Clinical Ophthalmology

\author{
Benjamin J Connell ${ }^{1,2}$ \\ Jack X Kane ${ }^{2}$ \\ Rasik B Vajpayee $\mathbb{D}^{2-4}$ \\ 'Eye Surgery Associates, Melbourne, \\ Victoria, Australia; ${ }^{2}$ Corneal Unit, Royal \\ Victorian Eye and Ear Hospital, \\ Melbourne, Victoria, Australia; ${ }^{3}$ Centre \\ for Eye Research Australia, University of \\ Melbourne, Melbourne, Victoria, \\ Australia; ${ }^{4}$ Vision Eye Institute, \\ Melbourne, Victoria, Australia
}

Correspondence: Benjamin J Connell Eye Surgery Associates, 2/232 Victoria Pde, East Melbourne, VIC, 3002, Australia Tel +6I 94160695

Fax +6194161816

Email benconnell@outlook.com.au
Purpose: To compare the refractive outcome prediction accuracy between conventional (CCS) and femtosecond laser assisted (FLACS) cataract surgery techniques using optimized lens constants for modern intraocular lens (IOL) formulas.

Patients and Methods: Our retrospective, comparative, interventional case series, compared data from 196 eyes undergoing CCS and 456 eyes undergoing FLACS with Acrysof IOL (Alcon laboratories, Inc) implantation. After optimizing IOL constants, the predicted refractive outcome was calculated for all formulas for each case. This was compared to the actual refractive outcome to provide the prediction error. The performance of CCS and FLACS was compared by the absolute prediction error and percentage of eyes within $0.25 \mathrm{D}, 0.5 \mathrm{D}$ and $1.0 \mathrm{D}$ of anticipated refractive outcome.

Results: There was no statistically significant difference in median absolute error between the CCS and LACS groups for the Kane (0.256, 0.236; p=0.389), SRK T $(0.298,0.302$, $\mathrm{p}=0.910)$, Holladay $(0.312,0.275 ; \mathrm{p}=0.090)$, Hoffer $\mathrm{Q}(0.314,0.289 ; \mathrm{p}=0.330)$, Haigis $(0.309,0.258 ; \mathrm{p}=0.177)$, Barrett Universal 2(0.250, 0.250; $\mathrm{p}=0.866)$, Holladay $2(0.250$, $0.258 ; \mathrm{p}=0.860)$ and Olsen $(0.260,0.255 ; \mathrm{p}=0.570)$ formulas. Similarly, there was no consistent difference between the two techniques for percentage of patients within 0.25 , 0.50 and $1.0 \mathrm{D}$ of predicted refractive outcome for each formula.

Conclusion: There was no difference in refractive outcome prediction accuracy between the CCS and FLACS techniques.

Keywords: femtosecond laser-assisted cataract surgery, refractive predictability, IOL formulas

\section{Introduction}

Recent generation of IOL formulas have significantly improved the refractive outcome prediction accuracy of modern cataract surgery. The ability of modern IOL formulas to achieve superior refractive outcomes over earlier third generation formulas $^{1-3}$ has been possible due to their accuracy at predicting the effective lens position. Recent large studies ${ }^{4-7}$ comparing CCS and FLACS have demonstrated no clear differences in prediction of refractive accuracy using earlier IOL formulas. However, none of these studies undertook the recommended ${ }^{8}$ IOL constant optimization to eliminate the source of bias inherent to IOL constants recommended by manufacturers.

We postulated that the improved precision of modern formulas, together with IOL constant optimization, may demonstrate that the superior capsulotomy 
geometry and IOL position ${ }^{9,10}$ advantages with FLACS translates into improved refractive outcomes.

In the present study, we investigated whether refractive outcome predictions were more accurate in FLACS when compared with CCS using optimized IOL constants for modern IOL formulas.

\section{Patients and Methods}

This retrospective, comparative, interventional comparative case series included all patients that had undergone CCS or FLACS surgery performed by a single surgeon (BC) between July 2015 and July 2019. Patients who had co- morbidities such as corneal scarring or previous ocular surgery were excluded. Other exclusion criteria were occurrence of intraoperative or postoperative complications or patients who had post-operative vision less than $6 / 12(20 / 40)$ equivalent.

If both eyes from a single patient met the inclusion criteria, one eye was randomly chosen for inclusion in the analysis. The study was approved by the Royal Victorian Eye and Ear Hospital Human Research Ethics Committee. Individual patient consent was not required as no patient identifying data was stored and retrospective study design, in compliance with the local data privacy laws. The study was conducted in compliance with the Declaration of Helsinki.

\section{Preoperative and Postoperative Examinations}

Patients underwent a preoperative full visual acuity assessment, slit lamp anterior and posterior segment examination. Preoperative biometry was performed using the IOLMaster model 700 (Carl Zeiss Meditec AG).

\section{Group Allocation}

The population typically held private health insurance and each patient self-selected either technique based on their own preference, considering the increased out of pocket expense for the laser assisted technique.

\section{Surgical Techniques}

The same surgeon (BC) performed all surgeries in a private operating facility under topical anesthesia. Capsulotomies were centered on the pupil. After removal of the cataract, an Alcon SN60WF or Alcon T6 series (Alcon Laboratories, Fort Worth Texas) IOL was injected. The wound was enlarged only for higher powered IOLs, as per the manufacturer's recommendation. Postoperative management was identical for the two groups. Prednisolone acetate 1\% (Prednefrin Forte, Allergan) and Chloramphenicol 0.5\% (Chlorsig, Sigma Pharmaceuticals, Australia) were used four times per day for 4 weeks following surgery.

\section{Conventional Technique}

In this technique, clear corneal temporal $2.4 \mathrm{~mm}$ wounds were used and the capsulotomy performed using forceps, followed by traditional phacoemulsification.

\section{Femtosecond Laser Technique}

The laser (LenSx platform, Alcon Surgical Inc) was used to perform the capsulotomy, lens fragmentation and wound construction. The pupil centered capsulotomy was used with the following parameters: $4.9 \mathrm{~mm}$ diameter, with delta up $270 \mu \mathrm{m}$, delta down $330 \mu \mathrm{m}$, spot energy 6.50 $\mu \mathrm{J}$, spot separation $4 \mu \mathrm{m}$ and layer separation $4 \mu \mathrm{m}$. Traditional phacoemulsification was then used to remove the nucleus.

\section{Formulas to Predict Post-Operative Spherical Equivalent Outcome} Haigis, ${ }^{11}$ Hoffer $Q,{ }^{12,13}$ Holladay ${ }^{14}$ and SRK/T ${ }^{15}$ formulas were programmed into a previously validated ${ }^{16}$ Excel spreadsheet (Microsoft, Redmond, Washington, USA) using the original publications and errata.

Data was entered into the respective third-party calculators for the other formulas: PhacoOptics program for the Olsen formula, ${ }^{17}$ IOL Consultant software for the Holladay 2 formula, ${ }^{18}$ and online calculators for the Barrett Universal 2. ${ }^{19}$ The Kane formula was calculated by one of the authors (JK).

The constant for each formula was optimized to produce a mean prediction error of zero (or as close as possible) by performing multiple iterations of the data using varying constants. For the Haigis formula, results were included for single $\left(a_{0}\right)$ and triple constant optimization.

For some formulas, a mean prediction error of zero could not be obtained due to limitations in how many decimal places could be entered for the constant into the calculator. In these cases the small residual mean error was removed by adjusting the refractive prediction error for each eye by an amount equal to the mean prediction error in that group as described in the JCRS editorial by Wang et al. ${ }^{20}$ 


\section{Statistical Analysis}

All statistical analysis was performed using Stata IC version 14 (College Station, TX, USA). Categorical variables were compared between surgical technique groups with Fisher's exact test. The distribution of continuous variables was assessed with the Shapiro-Wilk normality test and then the CCS and FLACS groups were compared using the twosample $t$-test for normally-distributed variables and the Wilcoxon rank-sum test if the variable was not normally distributed.

\section{Results}

During the study period 352 CCS and 826 FLACS procedures were performed. After exclusions, 196 eyes (from 196 patients) were included in the CCS group and 456 eyes (from 456 patients) in the FLACS group. Details of the exclusions are show in Table 1.

Small, statistically significant differences were noted in the median for baseline demographics between the two groups (Table 2). The CCS group was older (75 v 73 years; $\mathrm{p}=0.008)$, had shorter axial length (23.33 $\mathrm{v} 23.66 \mathrm{~mm} ; \mathrm{p}=0.028)$, anterior chamber depth $(3.03$ $\mathrm{v} 3.14 \mathrm{~mm} ; \mathrm{p}=0.002)$, thicker lens $(4.71 \mathrm{v} 4.59 \mathrm{~mm}$; $\mathrm{p}=<0.001)$ and inferior post-operative corrected distance visual acuity $(0.00 \mathrm{v} 0.00 ; \mathrm{p}=0.005)$.

There was no statistically significant difference in gender proportions (37/40\% Male) or toric IOL use (71\% for both) between the CCS/FLACS groups. The groups displayed similar distributions of certain comorbidities (Table 3). Optimized constants are shown in Table 4.

There was no statistically significant difference in the median absolute error between the CCS and FLACS groups for any of the formulas (Table 5). There was also no difference in percentage of patients within $0.25,0.50$ and $1.00 \mathrm{D}$ of predicted refraction for the CCS compared to the FLACS group (Table 6) for any of the formulas with the only exception the single constant optimized Haigis formula, where the percentage achieving within 1.0D of predicted was statistically higher in the FLACS group (98.5\% v 95.9\%; p=0.047).

\section{Discussion}

A femtosecond capsulotomy used in FLACS is considered geometrically superior to the manual capsulotomy in CCS. Studies have demonstrated it to have a more predictable diameter, ${ }^{9,21-24}$ more circular, ${ }^{9,21-25}$ less eccentric, ${ }^{9,23,24}$ and less shrinkage post-operatively. ${ }^{21,26}$ Consequently, the IOL position in FLACS demonstrates less tilt, ${ }^{10}$ decentration, ${ }^{10}$ greater overlap of the optic ${ }^{9}$ and the post-operative IOL anterior-posterior position deviates less from predicted. ${ }^{21}$

Effective lens position has been shown to be the most important factor in refractive prediction accuracy. ${ }^{27} \mathrm{We}$ hypothesized, that the superior FLACS capsulotomy geometry when compared with CCS, might translate improved in refractive accuracy by improving the predictability of the effective lens position.

Clinical studies to date have typically used 3rd generation formulas and not consistently demonstrated a refractive benefit for FLACS. The FEMCAT, ${ }^{4}$ FACT $^{5}$ and Roberts ${ }^{28}$ randomized control trials, a prospective intraindividual trial, $^{29}$ the EUREQUO registry ${ }^{30}$ and retrospective studies by Berk ${ }^{6}$ and Chee, ${ }^{7}$ have all used 3rd generation formulas and relatively large numbers have not demonstrated any refractive advantage. Ewe and colleagues, ${ }^{31}$ in a prospective, non-randomized comparative study demonstrated an advantage for CCS over FLACS.

Only a few smaller studies, also using 3rd generation formulas, have identified a refractive advantage for FLACS. A prospective study of 132 eyes $^{32}$ published in 2012 reported the mean absolute error was less with

Table I Indications for Subject Exclusion from the Analysis

\begin{tabular}{|l|l|l|l|l|}
\hline \multirow{2}{*}{ Exclusion Indication } & \multicolumn{2}{l|}{ CCS (352 Surgeries) } & \multicolumn{2}{l|}{ FLACS (826 Surgeries) } \\
\cline { 2 - 6 } & N Excluded & (Total Remaining) & N Excluded & (Total Remaining) \\
\hline Co-morbidities & 23 & $(329)$ & 67 & $(759)$ \\
Intra operative complication & 1 & $(328)$ & 2 & $(757)$ \\
Missing post-operative subjective refraction & 0 & $(328)$ & 2 & $(755)$ \\
Post-operative VA worse than 6/I2 & 6 & $(322)$ & 19 & $(736)$ \\
Missing biometry (unable to measure AXL or K's) & 0 & $(322)$ & 2 & $(734)$ \\
Random exclusion of I eye where both eyes eligible & 126 & $(196)$ & 278 & $(456)$ \\
\hline Final counts & 196 & & 456 & \\
\hline
\end{tabular}


Table 2 Baseline Demographic and Clinical Characteristics

\begin{tabular}{|c|c|c|c|}
\hline & CCS & FLACS & P value* \\
\hline \multicolumn{4}{|l|}{ Age $(y)$} \\
\hline Mean $(95 \% \mathrm{Cl})$ & $74.0(72.7,75.3)$ & $72.4(7 \mid .5,73.2)$ & \\
\hline Median (IQR) & $75.0(70.0,80.0)$ & $73.0(67.0,79.0)$ & 0.008 \\
\hline \multicolumn{4}{|l|}{ Gender } \\
\hline Male, n (\%) & $73(37.2)$ & $184(40.4)$ & 0.485 \\
\hline \multicolumn{4}{|c|}{ Pre op UDVA (logMAR) } \\
\hline Mean $(95 \% \mathrm{Cl})$ & $0.58(0.53,0.64)$ & $0.60(0.56,0.64)$ & \\
\hline Median (IQR) & $0.60(0.40,0.80)$ & $0.50(0.30,0.80)$ & 0.840 \\
\hline \multicolumn{4}{|c|}{ Pre op CDVA (logMAR) } \\
\hline Mean $(95 \% \mathrm{Cl})$ & $0.20(0.17,0.22)$ & $0.20(0.18,0.22)$ & \\
\hline Median (IQR) & $0.20(0.10,0.30)$ & $0.20(0.10,0.30)$ & 0.475 \\
\hline \multicolumn{4}{|l|}{ Axial length (mm) } \\
\hline Mean $(95 \% \mathrm{Cl})$ & $23.62(23.45,23.79)$ & $23.79(23.67,23.91)$ & \\
\hline Median (IQR) & $23.33(22.84,24.14)$ & $23.66(22.97,24.50)$ & $0.028^{\#}$ \\
\hline \multicolumn{4}{|c|}{ Anterior chamber depth (mm) } \\
\hline Mean $(95 \% \mathrm{Cl})$ & $3.05(3.00,3.11)$ & $3.15(3.11,3.18)$ & \\
\hline Median (IQR) & $3.03(2.77,3.29)$ & $3.14(2.88,3.39)$ & $0.002^{\#}$ \\
\hline \multicolumn{4}{|c|}{ Lens thickness (mm) } \\
\hline Mean $(95 \% \mathrm{Cl})$ & $4.70(4.64,4.76)$ & $4.59(4.55,4.63)$ & \\
\hline Median (IQR) & $4.71(4.42,4.99)$ & $4.59(4.29,4.87)$ & $<0.00 \mathrm{I}^{\#}$ \\
\hline \multicolumn{4}{|c|}{ Mean keratometry (D) } \\
\hline Mean $(95 \% \mathrm{Cl})$ & $43.92(43.70,44.14)$ & $43.79(43.65,43.93)$ & \\
\hline Median (IQR) & 43.91 (42.8I, 44.9I) & $43.75(42.75,44.89)$ & 0.393 \\
\hline \multicolumn{4}{|c|}{ Corneal astigmatism (D) } \\
\hline Mean $(95 \% \mathrm{Cl})$ & $0.96(0.86,1.05)$ & $0.89(0.83,0.96)$ & \\
\hline Median (IQR) & $0.81(0.50,1.28)$ & $0.76(0.43,1.10)$ & 0.091 \\
\hline \multicolumn{4}{|c|}{ Central corneal thickness $(\mu \mathrm{m})$} \\
\hline Mean $(95 \% \mathrm{Cl})$ & $548(543,553)$ & $553(549,556)$ & \\
\hline Median (IQR) & $546(527,565)$ & $553(529,574)$ & $0.044^{\#}$ \\
\hline \multicolumn{4}{|c|}{ Horizontal white to white $(\mathrm{mm})$} \\
\hline Mean $(95 \% \mathrm{Cl})$ & $11.92(11.85,11.98)$ & $11.98(11.94,12.02)$ & \\
\hline Median (IQR) & $11.90(11.65,12.30)$ & $12.00(11.70,12.30)$ & 0.281 \\
\hline \multicolumn{4}{|c|}{ Pupil diameter (mm) } \\
\hline Mean $(95 \% \mathrm{Cl})$ & $3.83(3.68,3.97)$ & $3.75(3.67,3.84)$ & \\
\hline Median (IQR) & $3.60(3.10,4.35)$ & $3.60(3.10,4.20)$ & 0.561 \\
\hline \multicolumn{4}{|c|}{ Post op UDVA (logMAR) } \\
\hline Mean $(95 \% \mathrm{Cl})$ & $0.28(0.25,0.32)$ & $0.27(0.24,0.29)$ & \\
\hline Median (IQR) & $0.20(0.10,0.40)$ & $0.20(0.10,0.40)$ & 0.274 \\
\hline \multicolumn{4}{|c|}{ Post op refractive astigmatism (D) } \\
\hline Mean $(95 \% \mathrm{Cl})$ & $0.42(0.36,0.47)$ & $0.39(0.36,0.42)$ & \\
\hline Median (IQR) & $0.50(0.00,0.50)$ & $0.50(0.00,0.50)$ & 0.475 \\
\hline \multicolumn{4}{|c|}{ Post op spherical equivalent (D) } \\
\hline Mean $(95 \% \mathrm{Cl})$ & $-0.91(-1.03,-0.79)$ & $-0.90(-0.98,-0.83)$ & \\
\hline Median (IQR) & $-0.75(-1.25,-0.38)$ & $-0.75(-1.25,-0.38)$ & 0.682 \\
\hline
\end{tabular}

(Continued) 
Table 2 (Continued).

\begin{tabular}{|l|l|l|l|}
\hline & CCS & FLACS & P value* \\
\hline $\begin{array}{l}\text { Post op CDVA (logMAR) } \\
\text { Mean (95\% Cl) }\end{array}$ & $0.02(0.00,0.03)$ & $-0.01(-0.02,0.00)$ & \\
Median (IQR) & $0.00(-0.10,0.10)$ & $0.00(-0.10,0.00)$ & $0.005^{\#}$ \\
\hline
\end{tabular}

Notes: *p value relates to the Wilcoxon Rank-Sum test for continuous variables and Fisher exact test for gender and IOL type. " Statistically significant difference between surgical technique groups.

Abbreviations: $\mathrm{Cl}$, confidence interval; IQR, interquartile range, UDVA, uncorrected distance visual acuity; CDVA, corrected distance visual acuity; D, dioptres.

Table 3 Comorbidity Counts

\begin{tabular}{|c|c|c|c|}
\hline Comorbidity & CCS & FLACS & P value* \\
\hline Amblyopia, n (\%) & $3(1.5)$ & $5(1.1)$ & 0.702 \\
\hline Fuchs endothelial dystrophy, n (\%) & $\mathrm{I}(0.5)$ & $13(2.9)$ & 0.076 \\
\hline Past pterygium surgery, n (\%) & $2(1.0)$ & $6(1.3)$ & 1.000 \\
\hline Current prostaglandin drop use, n (\%) & $10(5.1)$ & $21(4.6)$ & 0.841 \\
\hline Glaucoma involving fixation, $\mathrm{n}(\%)$ & $2(1.0)$ & $2(0.4)$ & 0.588 \\
\hline Pseudoexfoliation syndrome, n (\%) & $2(1.0)$ & $12(2.6)$ & 0.249 \\
\hline Oral alpha agonist use, n (\%) & $2(1.0)$ & $9(2.0)$ & 0.519 \\
\hline Past vitrectomy, n (\%) & 0 & $5(I . I)$ & 0.329 \\
\hline Past retinal detachment, $\mathrm{n}(\%)$ & 0 & $3(0.7)$ & 0.558 \\
\hline Epiretinal membrane, n (\%) & $5(2.6)$ & $13(2.9)$ & 1.000 \\
\hline Age related maculopathy, n (\%) & $17(8.7)$ & $47(10.3)$ & 0.569 \\
\hline Diabetes, n (\%) & $16(8.2)$ & $34(7.5)$ & 0.750 \\
\hline Diabetic retinopathy, n (\%) & $3(1.5)$ & I $(0.2)$ & 0.084 \\
\hline
\end{tabular}

Note: *p value relates to Fisher exact test for difference between surgical technique groups.

FLACS $(0.5 / 0.38 \mathrm{D}, \mathrm{p}=0.04)$. A prospective intraindividual study by Conrad-Hengerer ${ }^{33}$ with one eye of 100 patients randomized to each technique published in 2015 demonstrated $71 / 92 \%(p<0.05)$ of eyes within $0.5 \mathrm{D}$ of the intended outcome for CCS/FLACS. An accuracy of $92 \%$ of eyes achieving within $0.5 \mathrm{D}$ for FLACS

Table 4 Constants Used for the Different Formulas

\begin{tabular}{|l|l|l|l|}
\hline Formula & Constant & SN60WF & SN6ATx \\
\hline SRKT & & 118.72 & 118.91 \\
\hline Holladay I & & 1.63 & 1.77 \\
\hline Hoffer Q & & 5.41 & 5.56 \\
\hline Haigis single optimisation & a0 & -0.990 & -0.828 \\
& al & a2 & 0.234 \\
\hline Haigis triple optimisation & a0 & 0.217 & 0.217 \\
& al & -0.165 & -0.590 \\
\hline Barrett Universal 2 & a2 & 0.359 & 0.408 \\
\hline Olsen & & 0.166 & 0.184 \\
\hline Holladay 2 & ACD const. & 118.71 & 118.96 \\
\hline Kane & C & 4.43 & 4.56 \\
\hline
\end{tabular}


demonstrated in the Conrad-Hengerer study is relatively high and has not been replicated in other studies. For example, our study with modern biometry, optimized constants and strict case exclusion criteria demonstrated only $72 / 74 \%(\mathrm{SRK} / \mathrm{T})$ and 79/81\% (Haigis) from the CCS/ FLACS groups were within $0.5 \mathrm{D}$ of the intended outcome. Other large studies $5,6,28,31,34$ have typically reported $75 \%$ or less and no more than $83 \%$, in either group, achieving within $0.5 \mathrm{D}$ of the intended outcome.

More recent relatively small studies, also using older 3rd formulas, have only demonstrated a benefit for FLACS on some outcome measures. A recent retrospective study with 50 cases in each group, reported a significantly greater percentage of eyes within $0.5 \mathrm{D}$ of the intended outcome $(48 / 76 \%, p=0.01)$ but no difference between groups for the mean absolute error. In this study, there was a mean prediction error difference between the groups $(-0.42 /-0.11)$ because they did not optimize their lens constants. This difference in mean prediction error likely explains the difference in mean absolute errors reported. A large retrospective comparative case series with 3144 eyes $^{34}$ demonstrated a statistically significant lower mean absolute error for FLACS compared with CCS $(0.60$ $\mathrm{v} 0.54 \mathrm{D})$ however there was no difference in percentage of patients within $0.5 \mathrm{D}$ of intended.

None of these studies had used modern IOL formulas such as the Barrett Universal 2, Olsen or Kane formulas which have been reported to be more accurate than third generation

Table 5 Prediction and Absolute Errors for Each Formula

\begin{tabular}{|c|c|c|c|c|}
\hline & \multirow{2}{*}{$\begin{array}{l}\text { Prediction Error } \\
\text { All Eyes }\end{array}$} & \multicolumn{2}{|l|}{ Absolute Error } & \multirow[b]{2}{*}{$\mathbf{p}^{*}$} \\
\hline & & CCS & FLACS & \\
\hline \multicolumn{5}{|l|}{ Kane } \\
\hline Mean $(95 \% \mathrm{Cl})$ & $0.000(-0.029,0.029)$ & $0.312(0.277,0.347)$ & $0.289(0.268,0.309)$ & \\
\hline Median (IQR) & $0.021(-0.237,0.246)$ & $0.256(0.135,0.433)$ & $0.236(0.119,0.410)$ & 0.389 \\
\hline \multicolumn{5}{|l|}{ SRKT } \\
\hline Mean $(95 \% \mathrm{Cl})$ & $0.000(-0.035,0.035)$ & $0.362(0.322,0.403)$ & $0.363(0.337,0.389)$ & \\
\hline Median (IQR) & $0.007(-0.293,0.312)$ & $0.298(0.148,0.514)$ & $0.302(0.150,0.518)$ & 0.910 \\
\hline \multicolumn{5}{|l|}{ Holladay I } \\
\hline Mean $(95 \% \mathrm{Cl})$ & $0.000(-0.033,0.033)$ & $0.368(0.330,0.406)$ & $0.330(0.307,0.354)$ & \\
\hline Median (IQR) & $0.003(-0.280,0.290)$ & $0.312(0.165,0.505)$ & $0.275(0.136,0.460)$ & 0.090 \\
\hline \multicolumn{5}{|l|}{ Hoffer Q } \\
\hline Mean $(95 \% \mathrm{Cl})$ & $0.000(-0.034,0.034)$ & $0.373(0.334,0.413)$ & $0.344(0.321,0.368)$ & \\
\hline Median (IQR) & $0.014(-0.283,0.305)$ & $0.314(0.145,0.555)$ & $0.289(0.140,0.498)$ & 0.330 \\
\hline \multicolumn{5}{|l|}{ Haigis } \\
\hline Mean $(95 \% \mathrm{Cl})$ & $0.000(-0.03 \mathrm{I}, 0.03 \mathrm{I})$ & $0.344(0.306,0.382)$ & $0.308(0.286,0.330)$ & \\
\hline Median (IQR) & $0.006(-0.260,0.291)$ & $0.309(0.139,0.467)$ & $0.258(0.122,0.442)$ & 0.177 \\
\hline \multicolumn{5}{|l|}{ Haigis Triple } \\
\hline Mean $(95 \% \mathrm{Cl})$ & $0.000(-0.03 \mathrm{I}, 0.03 \mathrm{I})$ & $0.333(0.295,0.372)$ & $0.304(0.282,0.326)$ & \\
\hline Median (IQR) & $0.011(-0.259,0.273)$ & $0.272(0.128,0.464)$ & $0.262(0.119,0.417)$ & 0.326 \\
\hline \multicolumn{5}{|l|}{ Barrett Univ.2 } \\
\hline Mean $(95 \% \mathrm{Cl})$ & $0.000(-0.030,0.030)$ & $0.314(0.276,0.352)$ & $0.300(0.278,0.321)$ & \\
\hline Median (IQR) & $0.015(-0.232,0.253)$ & $0.250(0.122,0.435)$ & $0.250(0.110,0.425)$ & 0.866 \\
\hline \multicolumn{5}{|l|}{ Holladay 2} \\
\hline Mean $(95 \% \mathrm{Cl})$ & $0.000(-0.029,0.032)$ & $0.316(0.280,0.352)$ & $0.307(0.285,0.329)$ & \\
\hline Median (IQR) & $0.007(-0.240,0.263)$ & $0.250(0.132,0.430)$ & $0.258(0.132,0.415)$ & 0.860 \\
\hline \multicolumn{5}{|l|}{ Olsen } \\
\hline Mean $(95 \% \mathrm{Cl})$ & $-0.002(-0.033,0.028)$ & $0.320(0.284,0.355)$ & $0.303(0.281,0.324)$ & \\
\hline Median (IQR) & $-0.003(-0.250,0.260)$ & $0.260(0.115,0.440)$ & $0.255(0.130,0.420)$ & 0.570 \\
\hline
\end{tabular}

Note: ${ }^{*} \mathrm{p}$ value relates to the Wilcoxon rank-sum test for difference between groups.

Abbreviations: $\mathrm{Cl}$, confidence interval; $\mathrm{IQR}$, interquartile range. 
Table $6 \%$ of Eyes Within $0.25,0.50$ and I.0D of Absolute Error

\begin{tabular}{|c|c|c|c|}
\hline & CCS & FLACS & $\mathbf{P} *$ \\
\hline $\begin{array}{l}\text { Kane } \\
\leq 0.25 \mathrm{D}(95 \% \mathrm{Cl}) \\
\leq 0.5 \mathrm{D}(95 \% \mathrm{Cl}) \\
\leq 1.0 \mathrm{D}(95 \% \mathrm{Cl})\end{array}$ & $\begin{array}{l}49.5 \%(42.5,56.5) \\
81.6 \%(76.2,87.1) \\
98.0 \%(96.0,99.9)\end{array}$ & $\begin{array}{l}54.0 \%(49.4,58.5) \\
84.4 \%(81.1,87.8) \\
99.1 \%(98.3,100.0)\end{array}$ & $\begin{array}{l}0.296 \\
0.377 \\
0.216\end{array}$ \\
\hline $\begin{array}{l}\text { SRKT } \\
\leq 0.25 \mathrm{D}(95 \% \mathrm{Cl}) \\
\leq 0.5 \mathrm{D}(95 \% \mathrm{Cl}) \\
\leq 1.0 \mathrm{D}(95 \% \mathrm{Cl})\end{array}$ & $\begin{array}{l}39.8 \%(32.9,46.7) \\
71.9 \%(65.7,78.2) \\
96.4 \%(93.8,99.0)\end{array}$ & $\begin{array}{l}41.0 \%(36.5,45.5) \\
73.9 \%(69.9,77.9) \\
96.5 \%(94.8,98.2)\end{array}$ & $\begin{array}{l}0.772 \\
0.603 \\
0.968\end{array}$ \\
\hline $\begin{array}{l}\text { Holladay I } \\
\leq 0.25 \mathrm{D}(95 \% \mathrm{Cl}) \\
\leq 0.5 \mathrm{D}(95 \% \mathrm{Cl}) \\
\leq 1.0 \mathrm{D}(95 \% \mathrm{Cl})\end{array}$ & $\begin{array}{l}39.8 \%(32.9,46.7) \\
74.0 \%(67.8,80.1) \\
96.9 \%(94.5,99.4)\end{array}$ & $\begin{array}{l}43.2 \%(38.7,47.8) \\
79.0 \%(75.2,82.7) \\
98.0 \%(96.8,99.3)\end{array}$ & $\begin{array}{l}0.419 \\
0.164 \\
0.396\end{array}$ \\
\hline $\begin{array}{l}\text { Hoffer Q } \\
\leq 0.25 \mathrm{D}(95 \% \mathrm{Cl}) \\
\leq 0.5 \mathrm{D}(95 \% \mathrm{Cl}) \\
\leq 1.0 \mathrm{D}(95 \% \mathrm{Cl})\end{array}$ & $\begin{array}{l}43.4 \%(36.4,50.3) \\
68.9 \%(62.4,75.4) \\
97.5 \%(95.2,99.7)\end{array}$ & $\begin{array}{l}43.6 \%(39.1,48.2) \\
75.2 \%(71.3,79.2) \\
98.0 \%(96.8,99.3)\end{array}$ & $\begin{array}{l}0.949 \\
0.093 \\
0.641\end{array}$ \\
\hline $\begin{array}{l}\text { Haigis } \\
\leq 0.25 \mathrm{D}(95 \% \mathrm{Cl}) \\
\leq 0.5 \mathrm{D}(95 \% \mathrm{Cl}) \\
\leq 1.0 \mathrm{D}(95 \% \mathrm{Cl})\end{array}$ & $\begin{array}{l}42.4 \%(35.4,49.3) \\
79.1 \%(73.4,84.8) \\
95.9 \%(93.2,98.7)\end{array}$ & $\begin{array}{l}48.7 \%(44.1,53.3) \\
80.9 \%(77.3,84.5) \\
98.5 \%(97.3,99.6)\end{array}$ & $\begin{array}{l}0.137 \\
0.588 \\
0.047\end{array}$ \\
\hline $\begin{array}{l}\text { Haigis Triple } \\
\leq 0.25 \mathrm{D}(95 \% \mathrm{Cl}) \\
\leq 0.5 \mathrm{D}(95 \% \mathrm{Cl}) \\
\leq 1.0 \mathrm{D}(95 \% \mathrm{Cl})\end{array}$ & $\begin{array}{l}45.9(38.9,52.9) \\
80.6 \%(75.1,86.2) \\
96.9 \%(94.5,99.4)\end{array}$ & $\begin{array}{l}48.0 \%(43.4,52.6) \\
81.4 \%(77.8,84.9) \\
98.5 \%(97.3,99.6)\end{array}$ & $\begin{array}{l}0.621 \\
0.823 \\
0.201\end{array}$ \\
\hline $\begin{array}{l}\text { Barrett Univ. } 2 \\
\leq 0.25 \mathrm{D}(95 \% \mathrm{Cl}) \\
\leq 0.5 \mathrm{D}(95 \% \mathrm{Cl}) \\
\leq 1.0 \mathrm{D}(95 \% \mathrm{Cl})\end{array}$ & $\begin{array}{l}51.0 \%(44.0,58.0) \\
80.6 \%(75.1,86.2) \\
97.5 \%(95.2,99.7)\end{array}$ & $\begin{array}{l}51.5 \%(47.0,56.1) \\
82.0 \%(78.5,85.5) \\
98.3 \%(97.0,99.5)\end{array}$ & $\begin{array}{l}0.904 \\
0.671 \\
0.505\end{array}$ \\
\hline $\begin{array}{l}\text { Holladay } 2 \\
\leq 0.25 \mathrm{D}(95 \% \mathrm{Cl}) \\
\leq 0.5 \mathrm{D}(95 \% \mathrm{Cl}) \\
\leq 1.0 \mathrm{D}(95 \% \mathrm{Cl})\end{array}$ & $\begin{array}{l}50.5 \%(43.5,57.5) \\
80.1 \%(74.5,85.7) \\
96.9 \%(94.5,99.4)\end{array}$ & $\begin{array}{l}49.8 \%(45.2,54.4) \\
82.5 \%(79.0,86.0) \\
98.7 \%(97.6,99.7)\end{array}$ & $\begin{array}{l}0.864 \\
0.476 \\
0.128\end{array}$ \\
\hline $\begin{array}{l}\text { Olsen } \\
\leq 0.25 \mathrm{D}(95 \% \mathrm{Cl}) \\
\leq 0.5 \mathrm{D}(95 \% \mathrm{Cl}) \\
\leq 1.0 \mathrm{D}(95 \% \mathrm{Cl})\end{array}$ & $\begin{array}{l}49.0 \%(42.0,56.0) \\
79.6 \%(74.085 .2) \\
98.0 \%(96.0,99.9)\end{array}$ & $\begin{array}{l}49.6 \%(45.0,54.2) \\
81.8 \%(78.3,85.3) \\
98.7 \%(97.6,99.7)\end{array}$ & $\begin{array}{l}0.892 \\
0.509 \\
0.490\end{array}$ \\
\hline
\end{tabular}

Note: *p value relates to difference in proportions between groups.

Abbreviations: $\mathrm{Cl}$, confidence interval; $\mathrm{D}$, dioptres.

formulas ${ }^{1-3}$ to predict refractive accuracy. To the best of our knowledge, the only study using a later generation formula, was an intraindividual RCT of 110 paired eyes by Dzhaber ${ }^{35}$ which used the Holladay 2 formula and did not demonstrate any difference. With this formula they reported similar values to other studies with $81 / 84 \%(\mathrm{p}=0.17)$ of cases within $0.5 \mathrm{D}$ of the anticipated outcome. We hypothesized that when these modern formulas are used, there may be a refractive benefit for FLACS not evident with third generation formulas.
Ours is the first study that compared refractive prediction outcomes of FLACS and CCS using IOL constant optimization and included only one eye per patient as per the published recommendation. ${ }^{8}$ The recommendation to perform IOL constant optimization allows a more reliable comparison between formulas, with any difference in absolute error likely to reflect a true difference in formula accuracy. However, despite optimizing IOL constants in our study, the refractive accuracy was very similar to large prospective FEMCAT $^{4}$ and FACT $^{5}$ trials which did not perform optimization. Results of our study reconfirms that although the capsulotomy performed by FLACS may appear much more central and circular as compared to CCS, it does not translate in to better ELP and achieving more accurate refractive outcomes.

FLACS cases have also been demonstrated to show less capsular bag ${ }^{26}$ and capsulotomy shrinkage ${ }^{21}$ at $1-3$ months. It is also possible that these longer-term shrinkage forces are distributed relatively less symmetrically to the more irregular, less centered CCS capsulotomy and therefore more likely to induce long term IOL tilt, decentration and refractive change. This may be beneficial in the longer term for patients with multifocal and extended depth of focus lenses where lens tilt and decentration would degrade the visual outcome. A study using these lenses with longer term follow up would help address this question. A recent meta-analysis and commentary ${ }^{36,37}$ reported a clinically but not statistically significant lower rate of posterior capsule rupture with LACS, which would also benefit refractive outcome since IOL position is less predictable in these cases.

Overall, our study did not find any refractive advantage for FLACS over CCS when using modern IOL formulas and optimized IOL constants during a short-term follow-up. This confirms that a refractive advantage should not be used in guiding a patient's decision to proceed with either technique.

A disadvantage of our study is the potential for bias associated with patient self-selection for the either procedure. FLACS incurs an increased patient out of pocket expense in Australia of \$AUD850 (equivalent to \$US550 or 500euros).

The strengths of this study include that the surgeries were performed by a single surgeon, consistent staff performed the follow up assessments and modern biometry (IOLMaster model 700) was used. In addition, few patients were lost to follow up, the series was relatively large, and a systematic approach taken to case exclusion. 


\section{Conclusion}

Our study found no difference in refractive outcome prediction accuracy between the CCS and FLACS techniques using modern IOL formulas and optimized constants.

\section{Acknowledgments}

Sophie Rogers provided statistical support.

\section{Disclosure}

Jack X. Kane is the owner of the Kane formula. The authors report no other conflicts of interest in this work.

\section{References}

1. Melles RB, Holladay JT, Chang WJ. Accuracy of intraocular lens calculation formulas. Ophthalmology. 2018;125:169-178. doi:10.1016/j.ophtha.2017.08.027

2. Melles RB, Kane JX, Olsen T, Chang WJ. Update on intraocular lens calculation formulas. Ophthalmology. 2019;126:1334-1335. doi:10.1016/j.ophtha.2019.04.011

3. Darcy K, Gunn D, Tavassoli S, Sparrow J, Kane JX. Assessment of the accuracy of new and updated intraocular lens power calculation formulas in 10930 eyes from the UK National Health Service. $J$ Cataract Refract Surg. 2020;46:2-7. doi:10.1016/j.jcrs.2019.08.014

4. Schweitzer C, Brezin A, Cochener B, et al. Femtosecond laser-assisted versus phacoemulsification cataract surgery (FEMCAT): a multicentre participant-masked randomised superiority and cost-effectiveness trial. Lancet. 2020;395:212-224. doi:10.1016/ S0140-6736(19)32481-X

5. Day AC, Burr JM, Bennett K, et al. Femtosecond laser-assisted cataract surgery versus phacoemulsification cataract surgery (FACT): a randomized noninferiority trial. Ophthalmology. 2020;127:1012-1019. doi:10.1016/j.ophtha.2020.02.028

6. Berk TA, Schlenker MB, Campos-Moller X, Pereira AM, Ahmed IIK. Visual and refractive outcomes in manual versus femtosecond laser-assisted cataract surgery: a single-center retrospective cohort analysis of 1838 eyes. Ophthalmology. 2018;125:1172-1180. doi:10.1016/j.ophtha.2018.01.028

7. Chee SP, Yang Y, Ti SE. Clinical outcomes in the first two years of femtosecond laser-assisted cataract surgery. Am J Ophthalmol. 2015;159:714-719. doi:10.1016/j.ajo.2015.01.016

8. Hoffer KJ, Aramberri J, Haigis W, et al. Protocols for studies of intraocular lens formula accuracy. Am J Ophthalmol. 2015;160:403405 e1. doi:10.1016/j.ajo.2015.05.029

9. Nagy ZZ, Kranitz K, Takacs AI, Mihaltz K, Kovacs I, Knorz MC. Comparison of intraocular lens decentration parameters after femtosecond and manual capsulotomies. J Refract Surg. 2011;27:564-569. doi:10.3928/1081597X-20110607-01

10. Kranitz K, Mihaltz K, Sandor GL, Takacs A, Knorz MC, Nagy ZZ. Intraocular lens tilt and decentration measured by Scheimpflug camera following manual or femtosecond laser-created continuous circular capsulotomy. J Refract Surg. 2012;28:259-263. doi:10.3928/ 1081597X-20120309-01

11. Haigis W, Lege B, Miller N, Schneider B. Comparison of immersion ultrasound biometry and partial coherence interferometry for intraocular lens calculation according to Haigis. Graefes Arch Clin Exp Ophthalmol. 2000;238:765-773. doi:10.1007/s004170000188

12. Hoffer KJ. The Hoffer Q formula: a comparison of theoretic and regression formulas. J Cataract Refract Surg. 1993;19:700-712. doi:10.1016/S0886-3350(13)80338-0
13. Zuberbuhler B, Morrell AJ. Errata in printed Hoffer Q formula. J Cataract Refract Surg. 2007;33:2; author reply 2-3. doi:10.1016/j. jcrs.2006.08.054

14. Holladay JT, Prager TC, Chandler TY, Musgrove KH, Lewis JW, Ruiz RS. A three-part system for refining intraocular lens power calculations. J Cataract Refract Surg. 1988;14:17-24. doi:10.1016/ S0886-3350(88)80059-2

15. Retzlaff JA, Sanders DR, Kraff MC. Development of the SRK/T intraocular lens implant power calculation formula. $J$ Cataract Refract Surg. 1990;16:333-340. doi:10.1016/S0886-3350(13)80705-5

16. Kane JX, Van Heerden A, Atik A, Petsoglou C. Intraocular lens power formula accuracy: comparison of 7 formulas. $J$ Cataract Refract Surg. 2016;42:1490-1500. doi:10.1016/j.jcrs.2016.07.021

17. Olsen T. PhacoOptics. Available from: https://www.phacooptics.net/. Accesed October 1, 2019.

18. Holladay JT. Holladay IOL Consultant Software \& Surgical Outcomes Assessment. Bellaire, TX: Holladay Consulting; 2015.

19. Barrett GD. Barrett Universal II Formula. Singapore: Asia-Pacific Association of Cataract and Refractive Surgeons; 2018.

20. Wang L, Koch DD, Hill W, Abulafia A. Pursuing perfection in intraocular lens calculations: III. Criteria for analyzing outcomes. $J$ Cataract Refract Surg. 2017;43:999-1002. doi:10.1016/j. jcrs.2017.08.003

21. Panthier C, Costantini F, Rigal-Sastourne JC, et al. Change of capsulotomy over 1 year in femtosecond laser-assisted cataract surgery and its impact on visual quality. $J$ Refract Surg. 2017;33:44-49. doi:10.3928/1081597X-20161028-01

22. Friedman NJ, Palanker DV, Schuele G, et al. Femtosecond laser capsulotomy. J Cataract Refract Surg. 2011;37:1189-1198. doi:10.1016/j.jcrs.2011.04.022

23. Mastropasqua L, Toto L, Mattei PA, et al. Optical coherence tomography and 3-dimensional confocal structured imaging system-guided femtosecond laser capsulotomy versus manual continuous curvilinear capsulorhexis. J Cataract Refract Surg. 2014;40:2035-2043. doi:10.1016/j.jcrs.2014.05.032

24. Kranitz K, Takacs A, Mihaltz K, Kovacs I, Knorz MC, Nagy ZZ. Femtosecond laser capsulotomy and manual continuous curvilinear capsulorrhexis parameters and their effects on intraocular lens centration. $J$ Refract Surg. 2011;27:558-563. doi:10.3928/ 1081597X-20110623-03

25. Ostovic M, Klaproth OK, Hengerer FH, Mayer WJ, Kohnen T. Light microscopy and scanning electron microscopy analysis of rigid curved interface femtosecond laser-assisted and manual anterior capsulotomy. J Cataract Refract Surg. 2013;39:1587-1592. doi:10.1016/j.jcrs.2013.07.024

26. Dick HB, Conrad-Hengerer I, Schultz T. Intraindividual capsular bag shrinkage comparing standard and laser-assisted cataract surgery. $J$ Refract Surg. 2014;30:228-233. doi:10.3928/1081597X-201403 20-01

27. Norrby S. Sources of error in intraocular lens power calculation. J Cataract Refract Surg. 2008;34:368-376. doi:10.1016/j.jcrs.20 07.10.031

28. Roberts HW, Wagh VK, Sullivan DL, et al. A randomized controlled trial comparing femtosecond laser-assisted cataract surgery versus conventional phacoemulsification surgery. J Cataract Refract Surg. 2019;45:11-20. doi:10.1016/j.jcrs.2018.08.033

29. Krarup T, Ejstrup R, Mortensen A, la Cour M, Holm LM. Comparison of refractive predictability and endothelial cell loss in femtosecond laser-assisted cataract surgery and conventional phaco surgery: prospective randomised trial with 6 months of follow-up. BMJ Open Ophthalmol. 2019;4:e000233. doi:10.1136/bmjophth-2018-000233

30. Manning S, Barry P, Henry Y, et al. Femtosecond laser-assisted cataract surgery versus standard phacoemulsification cataract surgery: study from the European Registry of Quality Outcomes for Cataract and Refractive Surgery. $J$ Cataract Refract Surg. 2016;42:1779-1790. doi:10.1016/j.jcrs.2016.10.013 
31. Ewe SY, Abell RG, Oakley CL, et al. A comparative cohort study of visual outcomes in femtosecond laser-assisted versus phacoemulsification cataract surgery. Ophthalmology. 2016;123:178-182. doi:10.1016/j.ophtha.2015.09.026

32. Filkorn T, Kovacs I, Takacs A, Horvath E, Knorz MC, Nagy ZZ. Comparison of IOL power calculation and refractive outcome after laser refractive cataract surgery with a femtosecond laser versus conventional phacoemulsification. J Refract Surg. 2012;28:540-544. doi:10.3928/1081597X-20120703-04

33. Conrad-Hengerer I, Al Sheikh M, Hengerer FH, Schultz T, Dick HB. Comparison of visual recovery and refractive stability between femtosecond laser-assisted cataract surgery and standard phacoemulsification: six-month follow-up. $J$ Cataract Refract Surg. 2015;41:1356-1364. doi:10.1016/j.jcrs.2014.10.044

34. Nithianandan $\mathrm{H}$, Jegatheeswaran V, Dalal V, et al. Refractive laser-assisted cataract surgery vs. conventional manual surgery: comparing efficacy and safety in 3144 eyes. Am J Ophthalmol. 2019.
35. Dzhaber D, Mustafa OM, Alsaleh F, Daoud YJ, Daoud YJ. Visual and refractive outcomes and complications in femtosecond laser-assisted versus conventional phacoemulsification cataract surgery: findings from a randomised, controlled clinical trial. $\mathrm{Br}$ J Ophthalmol. 2020;104:225-229. doi:10.1136/bjophthalmol-2018313723

36. Kolb CM, Shajari M, Mathys L, et al. Comparison of femtosecond laser-assisted cataract surgery and conventional cataract surgery: a meta-analysis and systematic review. $J$ Cataract Refract Surg. 2020;46:1075-1085. doi:10.1097/j.jcrs.0000000000000228

37. Levitz LM, Wendell JS, Lawless M, Dick HB, Nagy Z. Comment on: comparison of femtosecond laser-assisted cataract surgery and conventional cataract surgery and conventional cataract surgery: a meta-analysis and systematic review. $J$ Cataract Refract Surg. 2021;47:278. doi:10.1097/j.jcrs.0000000000000534
Clinical Ophthalmology

\section{Publish your work in this journal}

Clinical Ophthalmology is an international, peer-reviewed journal covering all subspecialties within ophthalmology. Key topics include: Optometry; Visual science; Pharmacology and drug therapy in eye diseases; Basic Sciences; Primary and Secondary eye care; Patient Safety and Quality of Care Improvements. This journal is indexed on PubMed

Submit your manuscript here: https://www.dovepress.com/clinical-ophthalmology-journal
Dovepress

Central and CAS, and is the official journal of The Society of Clinical Ophthalmology (SCO). The manuscript management system is completely online and includes a very quick and fair peer-review system, which is all easy to use. Visit http://www.dovepress.com/ testimonials.php to read real quotes from published authors. 